The University of Southern Mississippi

The Aquila Digital Community

Faculty Publications

$9-1-2004$

\title{
On the Approximation of the Coriolis Terms in C-grid Models
}

Dmitri A. Nechaev

University of Southern Mississippi, dmitri.nechaev@usm.edu

Max Yaremchuk

University of Hawaii, maxy@soest.hawaii.edu

Follow this and additional works at: https://aquila.usm.edu/fac_pubs

Part of the Marine Biology Commons

\section{Recommended Citation}

Nechaev, D. A., Yaremchuk, M. (2004). On the Approximation of the Coriolis Terms in C-grid Models.

Monthly Weather Review, 132(9), 2283-2289.

Available at: https://aquila.usm.edu/fac_pubs/3023

This Article is brought to you for free and open access by The Aquila Digital Community. It has been accepted for inclusion in Faculty Publications by an authorized administrator of The Aquila Digital Community. For more information, please contact Joshua.Cromwell@usm.edu. 


\title{
On the Approximation of the Coriolis Terms in C-Grid Models
}

\author{
DMITRI NECHAEV \\ Department of Marine Science, University of Southern Mississippi, Stennis Space Center, Mississippi \\ MAX YAREMCHUK \\ International Pacific Research Center, University of Hawaii at Manoa, Honolulu, Hawaii
}

27 June 2003 and 19 February 2004

\section{ABSTRACT}

\begin{abstract}
A new representation of the Coriolis terms on the Arakawa $\mathrm{C}$ grid is proposed. The approximation dumps the grid-scale noise that arises because of spatial averaging of the Coriolis terms when the grid spacing is larger than the deformation radius. The proposed approximation can also be applied in C-grid schemes with semiimplicit treatment of the Coriolis terms. The new scheme is analyzed in the context of the linear inertial-gravity waves and its advantageous behavior is demonstrated with respect to the conventional technique.
\end{abstract}

\section{Introduction}

An important class of numerical models of ocean circulation are the so-called C-grid models [in the nomenclature of Arakawa and Lamb (1977)]. Because of the numerous well-known advantages of the C-grid discretization at high spatial resolutions, many ocean models have been developed on the horizontal C grid (e.g., Smith et al. 1990; Marshall et al. 1997; Madec et al. 1999). The $C$ grid is well suited for reproducing highfrequency inertia-gravity waves, but the conventional approximation of the primitive equations on the $\mathrm{C}$ grid suffer from certain difficulties in dealing with the Coriolis terms and low-frequency processes (Winninghoff 1968; Arakawa and Lamb 1977; Delecluse and Zalesnyy 1996; Adcroft et al. 1999). In particular, the C-grid approach is prone to grid-scale noise when the horizontal grid spacing $\delta x$ is larger than the deformation radius $R$; that is, when the resolution parameter $r=2 R / \delta x$ is low. The grid-scale noise can persist because of horizontal averaging of the velocity components that are staggered in space on the $\mathrm{C}$ grid. Although this type of noise has negligible projection on the eigenfunctions of linearized model equations in the unbounded ocean, it may eventually grow near sharp topographic features and affect the structure of the model fields, especially of the ver-

Corresponding author address: Max Yaremchuk, International Pacific Research Center, University of Hawaii at Manoa, 1680 East West Rd., POST Bldg. 401, Honolulu, HI 96822.

E-mail: maxy@soest.hawaii.edu tical velocity. The spatial four-point averaging of the Coriolis terms utilized in conventional C-grid schemes may lead to the excitation of a "checkerboard" noise in horizontal divergence and sea surface height ( $\mathrm{SSH}$ ) fields and, in some cases, to production of work by Coriolis terms. Averaging of the Coriolis terms also complicates their implicit representation, which is in many cases important for numerical stability.

This problem with the $\mathrm{C}$ grid has been known for a long time. A large number of "fixes," which basically employ scale-selective dissipation to dump grid-scale variability, have been proposed. Recently, Adcroft et al. (1999) suggested augmenting the C-grid horizontal velocities with $\mathrm{D}$-grid velocity variables. They have shown that such a hybrid C-/D-grid approach improved spectral properties of the inertia-gravity waves at low resolutions. This was achieved, however, at the expense of doubling the number of velocity variables.

In this note we propose an approach that does not require introduction of any additional degrees of freedom into the model. To obtain consistent velocity fields after one-step integration, we first find solutions to the original C-grid finite-difference equations with noncollocated velocities and then average these solutions to compute the divergence and eliminate the impact of spatial shifts on the final velocity field. The approach yields good results at both high and low resolutions and can be readily used in semi-implicit schemes. To illustrate the method, in the next section we briefly overview the conventional spatial averaging scheme and discuss 
the approach of Adcroft et al. (1999). In section 3 we give a detailed description of the numerical scheme, analyze its dispersion properties, and test its performance against the well-known community Ocean Parallelise (OPA) OGCM (Madec et al. 1999).

\section{C-grid representations of the inertia-gravity waves}

In this section we generally follow the treatment of Adcroft et al. (1999) who gave a comprehensive analysis of the "low-resolution C-grid problem." Consider linearized shallow-water equations on an infinite plane

$$
\begin{aligned}
& \partial_{t} \mathbf{u}=-f(\mathbf{k} \times \mathbf{u})-g \boldsymbol{\nabla} \zeta, \\
& \partial_{t} \zeta=-H \boldsymbol{\nabla} \cdot \mathbf{u},
\end{aligned}
$$

with ocean depth $H=$ const. Here $f$ is the Coriolis parameter, $\mathbf{u}$ is the horizontal velocity vector, $\zeta$ is the free surface elevation, and $g$ is the reduced gravity. Under this notation the Rossby deformation radius is $R=\sqrt{g H} / f$.

\section{a. Conventional technique}

The standard C-grid discretization with centered treatment of the Coriolis terms reads

$$
\begin{gathered}
\delta_{2 t} u-f \bar{v}^{x y}+g \delta_{x} \zeta=0, \\
\delta_{2 t} v+f \bar{u}^{x y}+g \delta_{y} \zeta=0, \\
\delta_{2 t} \zeta+H\left(\delta_{x} u+\delta_{y} v\right)=0 .
\end{gathered}
$$

Here, $\delta_{2 t}, \delta_{x}$, and $\delta_{y}$ are the centered discrete operators that approximate the continuous partial derivatives $\partial_{t}$, $\partial_{x}$, and $\partial_{y}$, respectively: $\delta_{2 t} \varphi=(1 / 2 \delta t)\left[\varphi^{t+\delta t}-\varphi^{t-\delta t}\right]$, $\delta_{x} \varphi=(1 / \delta x)\left[\varphi_{x+\delta x / 2}-\varphi_{x-\delta x / 2}\right], \delta_{y} \varphi=(1 / \delta y)\left[\varphi_{y+\delta y / 2}-\right.$ $\left.\varphi_{y-\delta y / 2}\right]$. The overbar denotes centered two-point averaging over the coordinates shown by the superscript: $\bar{\varphi}^{x}=(1 / 2)\left[\varphi_{x+\delta y / 2}+\varphi_{x-\delta y / 2}\right], \bar{\varphi}^{y}=(1 / 2)\left[\varphi_{y+\delta x / y / 2}+\right.$ $\left.\varphi_{y-\delta y / 2}\right]$. Substituting a plane wave solution $\exp [i(k x+$ $l y-\omega t)]$ into (1)-(3), one can get the dispersion relation in the form

$$
s_{\omega}\left[s_{\omega}^{2}-f^{2} c^{2}-g H\left(s_{k}^{2}+s_{l}^{2}\right)\right]=0,
$$

where $s_{\omega}=1 / \delta t \sin \omega \delta t, s_{k}=2 / \delta x \sin (k \delta x) / 2, s_{l}=$ $2 / \delta y \sin (l \delta y / 2)$, and $c=\cos (k \delta x / 2) \cos (l \delta y / 2)$. It is well known (e.g., Adcroft et al. 1999) that it is the factor $c^{2}$ that is responsible for excitation of the grid-scale noise when $\delta x>2 R$. In that case false minima appear in the $2 \mathrm{D}$ wavenumber space of the discrete representation of the analytic dispersion relation

$$
\omega\left[\omega^{2}-f^{2}-g H\left(k^{2}+l^{2}\right)\right]=0 .
$$

These minima reside near points $\{0, \pi / \delta y\},\{\pi / \delta x, 0\}$ and cause characteristic near-coastal noise with wave crests parallel to the coastlines and steep topographic features.

\section{b. C-/D-grid approach}

To bring the discrete dispersion relation closer to the analytic form (5) and eliminate false minima associated with spatial averaging, Adcroft et al. (1999) proposed to augment $\mathrm{C}$-grid model variables with $\mathrm{D}$-grid veloc- ities $\mathbf{u}^{d}$, such that zonal C-grid velocities $u^{c}$ are collocated with meridional D-grid velocities $v^{d}$ and vice versa. In their approach the factor $c$ is removed from the dispersion relation at the expense of two extra roots $\omega$ $= \pm 1 / \delta t \operatorname{atan} f \delta t$, which represent the computational modes. The latter emerge as a consequence of doubling the number of velocity variables on the C/D grid and have to be filtered. Adcroft et al. (1999) have shown that filtering could be effectively performed using implicit treatment of the Coriolis terms and can be readily implemented on the C/D grid because velocities $\mathbf{u}_{c}^{t+\delta t}$, $\mathbf{u}_{d}^{t+\delta t}$ can be expressed analytically in terms of $\zeta$. This is an important feature since Coriolis terms, when treated explicitly, may often cause numeric instabilities even at moderate time steps. In the conventional approach, velocities at $t+\delta t$ cannot be obtained analytically because of the nonlocal nature of spatial averaging.

An obvious disadvantage of the C-/D-grid approach is a substantial increase of the model's degrees of freedom and a necessity to filter the computational modes.

\section{Shifted approximations}

Delecluse and Zalesnyy (1996) analyzed approximations of linear shallow-water equations on $\mathrm{B}$ and $\mathrm{C}$ grids and noticed that the hybrid $\mathrm{C} / \mathrm{B}$ grid is capable of solving the problems caused by spatial averaging of the Coriolis terms. The paper also outlines the possibility of regularization of the C-grid approximation at low frequencies $\omega \ll f$ through "diagonalization" of the Coriolis terms. The simplest way to "diagonalize" the Coriolis term is to implement a "shifted" approximation:

$$
\begin{aligned}
& \delta_{2 t} u_{i-1 / 2, j}-f v_{i, j+1 / 2}=\cdots, \\
& \delta_{2 t} v_{i, j+1 / 2}+f u_{i-1 / 2, j}=\cdots .
\end{aligned}
$$

We call (6) the shifted approximation since $v$ is taken in the node that is displaced half a grid step in both directions of the horizontal coordinate axes with respect to the node, where the time derivative $\delta_{2 t} u$ is computed. The system (6) is pointwise (or diagonalized) with respect to $u_{i-1 / 2, j}$ and $v_{i, j+1 / 2}$ and can be easily solved if the scheme is implicit with respect to the Coriolis term.

\section{a. Numerical scheme}

In the following numerical treatment we propose to center the approximation (6) by averaging the result of time steppings over four possible shifted approximations:

$$
\mathbf{u}=\frac{1}{4}\left(\mathbf{u}_{1}+\mathbf{u}_{2}+\mathbf{u}_{3}+\mathbf{u}_{4}\right)=\frac{1}{4} \sum_{m=1}^{4} \mathbf{u}_{m},
$$

where $\mathbf{u}_{1}$ is the result of integration with the scheme (6), $\mathbf{u}_{2}$ is the velocity field obtained from the scheme, where $v$ is shifted in the negative direction in $x$ and positive in $y$ with respect to $u$ :

$$
\begin{aligned}
& \delta_{2 t} u_{i-1 / 2, j}-f v_{i-1, j+1 / 2}=\cdots, \\
& \delta_{2 t} v_{i-1, j+1 / 2}+f u_{i-1 / 2, j}=\cdots .
\end{aligned}
$$

Velocity fields $\mathbf{u}_{3}$ and $\mathbf{u}_{4}$ cover the remaining two of 
the four possible shift permutations. Introducing "shift operators" $\hat{\sigma}_{1}: \varphi_{x, y} \rightarrow \varphi_{x+1 / 2 \delta x, y+1 / 2 \delta y}, \hat{\sigma}_{2}: \varphi_{x, y} \rightarrow$ $\varphi_{x+1 / 2 \delta x, y-1 / 2 \delta y}$ etc., we may write down the symmetrized shifts approximation (SSA) scheme as follows:

$$
\begin{array}{cc}
\delta_{2 t} u_{m}-f \hat{\sigma}_{m} v_{m}+g \delta_{x} \zeta=0 & m=1, \ldots, 4 \\
\delta_{2 t} v_{m}+f \hat{\sigma}_{m}^{-1} u_{m}+g \delta_{y} \zeta=0 & m=1, \ldots, 4 \\
\delta_{2 t} \zeta+H\left(\delta_{x} u+\delta_{y} v\right)=0 . &
\end{array}
$$

Here the velocity field $\mathbf{u}$ in Eq. (11) is averaged over the four shifted approximations. As will be shown in the next section, the SSA scheme (9)-(11) resolves the grid-scale noise problem without introduction of the additional degrees of freedom into the velocity field since $\mathbf{u}_{m}$ are the same at the starting point of integration $t=$ 0 . An obvious disadvantage of the method is the necessity of carrying in time the four shifted solutions for the velocity field, which may be impractical and computationally inefficient. In an alternative approach, $\mathbf{u}_{m}$ can be reinitialized on every time step by taking their average. The resulting averaged symmetrized shifts approximation (ASSA) scheme is

$$
\begin{aligned}
\frac{1}{2 \delta t}\left[u_{m}^{t+\delta t}-u^{t-\delta t}\right]-f \hat{\sigma}_{m}\left[\alpha v_{m}^{t+\delta t}+\beta v^{t}+\gamma v^{t-\delta t}\right]+g \delta_{x} \zeta & =0 \quad m=1, \ldots, 4, \\
\frac{1}{2 \delta t}\left[v_{m}^{t+\delta t}-v^{t-\delta t}\right]+f \hat{\sigma}_{m}^{-1}\left[\alpha u_{m}^{t+\delta t}+\beta u^{t}+\gamma u^{t-\delta t}\right]+g \delta_{y} \zeta & =0 \quad m=1, \ldots, 4, \\
\mathbf{u}^{t+\delta t} & =\hat{A} \mathbf{u}_{m}^{t+\delta t}, \\
\delta_{2 t} \zeta+H\left(\delta_{x} u+\delta_{y} v\right) & =0 .
\end{aligned}
$$

Equations (12)-(15) are written for general implicit treatment of the Coriolis terms $(\alpha>0, \beta>0, \gamma>0$, $\alpha+\beta+\gamma=1)$. Here, $\hat{A}$ averages the shifted modes $\hat{A} \mathbf{u}_{m}=\mathbf{u}$, whereas its action is equivalent to four-point spatial averaging when $\hat{A}$ is applied to a single field taken with different shifts $\hat{A} \hat{\sigma}_{m} \mathbf{u}=\hat{A} \hat{\sigma}_{m}^{-1} \mathbf{u}=\overline{\mathbf{u}}^{x y}$. It is also obvious that $\hat{A} \mathbf{u} \equiv \mathbf{u}$. Application of $\hat{A}$ to Eqs. (12)-(13) results in the following relations:

$$
\begin{array}{r}
\delta_{2 t} u-f \bar{v}^{x y t}-\alpha f\left[\hat{A} \hat{\sigma}_{m} v_{m}^{t+\delta t}-\hat{A} \hat{\sigma}_{m} v^{t+\delta t}\right]+g \delta_{x} \zeta^{t}=0, \\
\delta_{2 t} v+f \bar{u}^{x y t}+\alpha f\left[\hat{A} \hat{\sigma}_{m}^{-1} u_{m}^{t+\delta t}-\hat{A} \hat{\sigma}_{m}^{-1} u^{t+\delta t}\right]+g \delta_{y} \zeta^{t}=0,
\end{array}
$$

where $\overline{\mathbf{u}}^{x y t}=\hat{A} \sigma_{m}\left(\alpha \mathbf{u}^{t+\delta t}+\beta \mathbf{u}^{t}+\gamma \mathbf{u}^{t-\delta t}\right)$. Equations (16)-(17) show that the numerical properties of the ASSA approximation depend on the particular form of the time averaging of the Coriolis term. For example, the explicit treatment of the Coriolis term $(\alpha=0)$ reduces the ASSA scheme to the standard C-grid approximation. On the other hand, in the case of $\alpha=1$, ASSA and implicit SSA schemes become identical. Note that numerically in both ASSA and SSA schemes the equations are first resolved with respect to $\mathbf{u}_{m}^{t+\delta t}$ and then averaged to obtain a single velocity field on the time layer $t+\delta t$. The only difference is that the ASSA scheme forgets the discrepancies between the shifted solutions $\mathbf{u}_{m}^{t+\delta t}$ after the time step is completed [there is a single velocity at times $t, t-\delta t$ in formulas (12)(13)], whereas the SSA scheme (9)-(11) keeps these solutions in memory to advance them further in time.
Implicit treatment of the pressure gradient terms in both ASSA and SSA schemes can be reduced to solution of a linear set of equations with respect to $\zeta^{t+\delta t}$, which have identical system matrices but different right-hand side vectors. Below we analyze numerical properties of the SSA and ASSA schemes with implicit treatment of both Coriolis and pressure gradient terms with centered time averaging $(\alpha=\gamma=1 / 2)$. To simplify further analysis, it is convenient to rewrite (12)-(15) in the equivalent form of three finite-difference equations that do not contain the shifted velocity fields. These relationships can be obtained by solving (12)-(13) with respect to $u_{m}^{t+\delta t}$, $v_{m}^{t+\delta t}$ and substituting the result into (16)-(17):

$$
\begin{array}{r}
\delta_{2 t} u-f \bar{v}^{x y t}+g \delta_{x} \bar{\zeta}^{t}+\varepsilon\left[\delta_{2 t} \bar{v}^{x y}+f \bar{u}^{t}+g{\overline{\delta_{y}} \zeta^{x y t}}^{x}=0\right. \\
\delta_{2 t} v+f \bar{u}^{x y t}+g \delta_{y} \bar{\zeta}^{t}-\varepsilon\left[\delta_{2 t} \bar{u}^{x y}-f \bar{v}^{t}+g{\overline{\delta_{x} \zeta}}^{x y t}\right]=0 \\
\delta_{2 t} \zeta+H\left(\delta_{x} \bar{u}^{t}+\delta_{y} \bar{v}^{t}\right)=0
\end{array}
$$

where $\varepsilon=f \delta t$. As it is seen from (18)-(20) in the lowfrequency limit $\left(\varepsilon \rightarrow \infty ; \delta t \gg f^{-1}\right)$ the ASSA scheme produces a geostrophic flow resulting from averaging of the four regular geostrophic velocity approximations $\mathbf{u}_{m}=g / f(\mathbf{k} \times \boldsymbol{\nabla}) \hat{\sigma}_{m} \zeta$. In the high-frequency limit $(\varepsilon \rightarrow$ $0)$, the scheme is equivalent to the conventional $\mathrm{C}$-grid approximation with implicit representation of the Coriolis terms. 


\section{b. Dispersion}

After substitution of the plane wave $\left\{\hat{U}_{m}, \hat{V}_{m}, \hat{\zeta}\right\}$ $\exp [i(k x+l y-\omega t)]$ into equations (9)-(11), they take the form

$$
\begin{array}{rc}
-i s_{\omega} \hat{U}_{m}-f c_{\omega} \mathrm{e}_{m} \hat{V}_{m}+i g s_{k} c_{\omega} \hat{\zeta}=0 & m=1, \ldots, 4 \\
f c_{\omega} \mathrm{e}_{m}^{-1} \hat{U}_{m}-i s_{\omega} \hat{V}_{m}+i g s_{l} c_{\omega} \hat{\zeta}=0 & m=1, \ldots, 4 \\
-s_{\omega} \hat{\zeta}+H c_{\omega}\left(s_{k} \hat{U}+s_{l} \hat{V}\right)=0 &
\end{array}
$$

where $c_{\omega}=\cos \omega \delta t$, and $\mathrm{e}_{1}=\exp [i(+k \delta x / 2+i \delta y / 2)]$, $\mathrm{e}_{2}=\exp [i(+k \delta x / 2-l \delta y / 2)]$ etc. are the Fourier images of the shift operators $\hat{\sigma}_{m}$. Amplitudes of the averaged Fourier components $\hat{U}, \hat{V}$ can be expressed in terms of $\hat{\zeta}$ using the solutions of (21)-(22) with respect to $\hat{U}_{m}$, $\hat{V}_{m}$ and taking into account that $c=1 / 4 \Sigma_{m} \mathrm{e}_{m}=1 / 4$ $\Sigma_{m} \mathrm{e}_{m}^{-1}$. By doing that one can obtain the dispersion relation for the SSA scheme in the form

$$
t_{\omega}\left[t_{\omega}^{2}-f^{2}-g H\left(s_{k}^{2}+s_{l}^{2}\right)\right]=0,
$$

where $t_{\omega}=s_{\omega} / c_{\omega}=1 / \delta t \tan \omega \delta t$. It is seen that the problematic factor $c^{2}$ in the Coriolis term [see Eq. (4)] is now absent. That is achieved, however, at the expense of the necessity to keep solutions of the four shifted velocity approximations.

The dispersion relation for the ASSA scheme can be obtained by applying a similar technique to Eqs. (18)(20). Substituting the plane wave and dividing (18)(20) by $-i c_{\omega}$, one can obtain

$$
\begin{aligned}
\bar{t}_{\omega} \hat{U}-i c \bar{f} \hat{V}-g\left(s_{k}+\varepsilon c s_{l}\right) \hat{\zeta} & =0, \\
i c \bar{f} \hat{U}+\overline{t_{\omega}} \hat{V}-g\left(s_{l}-\varepsilon c s_{k}\right) \hat{\zeta} & =0, \\
-H s_{k} \hat{U}-H s_{l} \hat{V}+t_{\omega} \hat{\zeta} & =0,
\end{aligned}
$$

where $\bar{t}_{\omega}=t_{\omega}+i \varepsilon f$, and $\bar{f}=f+i \varepsilon t_{\omega}$. The corresponding dispersion relation is

$$
t_{\omega}\left[{\overline{t_{\omega}}}^{2}-\bar{f}^{2} c^{2}\right]-g H\left(s_{k}^{2}+s_{l}^{2}\right)\left[\overline{t_{\omega}}-i \varepsilon \bar{f} c^{2}\right]=0 .
$$

At small time steps $(\varepsilon \rightarrow 0)$, the relationship (27) asymptotes to the version of (4) with semi-implicit Coriolis and pressure gradient terms.

In contrast to SSA, the ASSA dispersion relation contains complex-valued terms that may cause damping of the waves at certain wavenumbers. Consider this property at time steps $\delta t$, satisfying the temporal resolution condition $\omega \delta t \ll 0\left(t_{\omega} \approx \omega\right)$. At small, but finite $\varepsilon \rightarrow$ 0 the roots $\omega^{R}, \omega^{\mathrm{IG}}$, corresponding to Rossby (R) and inertia-gravity (IG) waves acquire small imaginary parts

$$
\begin{aligned}
& \operatorname{Im}\left(\omega^{R}\right)=-\varepsilon f\left(1-c^{2}\right) \frac{1}{1+\xi^{2}}+O\left(\varepsilon^{2}\right), \\
& \operatorname{Im}\left(\omega^{\mathrm{IG}}\right)=-\varepsilon f\left(1-c^{2}\right) \frac{1 / 2+\xi^{2}}{1+\xi^{2}}+O\left(\varepsilon^{2}\right),
\end{aligned}
$$

where $\xi^{2}=c^{2} /\left[R^{2}\left(s_{k}^{2}+s_{l}^{2}\right)\right]$ and $R^{2}=g H / f^{2}$. The sign of imaginary parts corresponds to damping of the waves, which is highly selective in wavenumbers because of the factor $\left(1-c^{2}\right)$. At wavelengths $\lambda \gg \delta x$ the factor becomes small since $1-c^{2} \sim \pi^{2} \delta x^{2} / \lambda^{2} \ll 1$. For wellresolved waves $s_{k, l} \ll 1, \xi \sim(k R)^{-1}$, and the Rossby waves with wavelengths $L \gg R$ have negligible damping. If, for example, $R=30 \mathrm{~km}, \delta x=10 \mathrm{~km}, f=10^{-4}$ $\mathrm{s}^{-1}$, and $\delta t=1000 \mathrm{~s}$, the Rossby waves with length $300 \mathrm{~km}$ will be damped with an $e$-folding time of approximately $1 \mathrm{yr}$. In the opposite limit of large time steps $\delta t \gg f^{-1}(\varepsilon \rightarrow \infty)$ the damping factor for the Rossby waves is

$$
\operatorname{Im}\left(\omega^{R}\right)=-\varepsilon^{-1} f\left(1-c^{2}\right) \frac{1}{c^{2}+\xi^{2}}+O\left(\varepsilon^{2}\right) .
$$

That is, for $\delta t=1$ day and the same wave/grid parameters as above, the damping scale will again be of the order of $1 \mathrm{yr}$.

\section{c. Energy conservation}

To simplify the derivation of energy equations we again consider the case of centered time averaging ( $\alpha$ $=\gamma=1 / 2$ ) and use the notation $M u=\bar{u}^{x y}$ for the fourpoint averaging operator. Consider an infinite $\mathrm{C}$ grid $C$ with gridded functions $u, v, \zeta$ taking nonzero values in some finite domain (ocean basin) that are extended with zeroes into the rigid boundaries. If we define the inner product $\langle a, b\rangle$ as pointwise summations of the appropriate fields over $\mathcal{C}$, then it can be shown that the grid operators $\delta_{x}, \delta_{y}, M$ have the following properties:

$$
\begin{aligned}
& \langle M a, b\rangle=\langle a, M b\rangle, \quad\left\langle\delta_{x} a, b\right\rangle=-\left\langle a, \delta_{x} b\right\rangle, \\
& \left\langle\delta_{y} a, b\right\rangle=-\left\langle a, \delta_{y} b\right\rangle,
\end{aligned}
$$

whereas $M$ also commutes with the differentiation operators

$$
M \delta_{x}-\delta_{x} M=M \delta_{y}-\delta_{y} M=0 .
$$

Consider first the quadratic form defined on the space of nine gridded fields $\left\{u_{m}, v_{m}, m=1, \ldots, 4 ; \zeta\right\}$

$$
\begin{aligned}
\mathbf{E}_{\mathrm{SSA}}\left\{u_{m}, v_{m}, \zeta\right\}= & \hat{A}\left[\left\langle\bar{u}_{m}^{t},(9)\right\rangle+\left\langle\bar{v}_{m}^{t},(10)\right\rangle\right] \\
& +\frac{g}{H}\left\langle\bar{\zeta}^{t},(11)\right\rangle,
\end{aligned}
$$

where numbers in brackets denote symbolically the lefthand sides of Eqs. (9)-(11). Using (28)-(29) the form can be reduced to the conservation equation

$$
\delta_{2 t}\left[\hat{A}\left(\left\langle u_{m}, u_{m}\right\rangle+\left\langle v_{m}, v_{m}\right\rangle\right)+\frac{g}{H}\langle\zeta, \zeta\rangle\right]=0,
$$

which shows that the SSA scheme conserves the sum of the potential energy and mean of the kinetic energies of the four shifted velocity fields.

Energy equation for the ASSA scheme can be derived by considering the form 

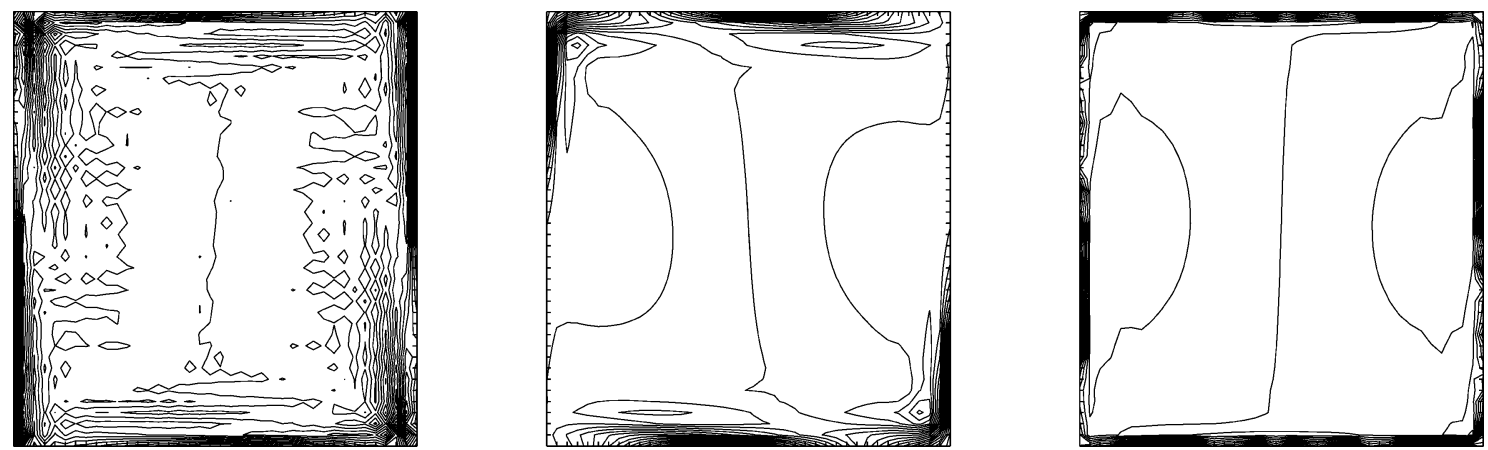

FIG. 1. Divergence fields after 50 days of integration of the (left) SEC, (middle) ASSA, and (right) SSA codes. Contour interval is $10^{-9} \mathrm{~s}^{-1}$.

$$
\begin{aligned}
\mathbf{E}_{\mathrm{ASSA}}\{u, v, \zeta\}= & \left.\left\langle\bar{u}^{t}+\varepsilon M \bar{v}^{t},(18)\right\rangle+\left\langle\bar{v}^{t}-\varepsilon M \bar{u}^{t},(19)\right\rangle\right\rangle \\
& +\frac{g}{H}\left[\left\langle\bar{\zeta}^{t},(20)\right\rangle+\varepsilon^{2}\left\langle M \bar{\zeta}^{t}, M(20)\right\rangle\right],
\end{aligned}
$$

where

$$
\begin{aligned}
\mathcal{E} & =\frac{1}{2}\left[\langle u, u\rangle+\langle v, v\rangle+\frac{g}{H}\langle\zeta, \zeta\rangle\right] ; \\
\mathcal{E}_{M} & =\frac{1}{2}\left[\langle M u, M u\rangle+\langle M v, M v\rangle+\frac{g}{H}\langle M \zeta, M \zeta\rangle\right]
\end{aligned}
$$

are the energies of the gridded $\mathcal{E}$ and the four-point averaged $\mathcal{E}_{M}$ fields, whereas $\mathcal{K}$ and $\mathcal{K}_{M}$ are the respective kinetic energies of the velocity fields

$$
\begin{aligned}
\mathcal{K} & =\frac{1}{2}\left[\left\langle\bar{u}^{t}, \bar{u}^{t}\right\rangle+\left\langle\bar{v}^{t}, \bar{v}^{t}\right\rangle\right], \\
\mathcal{K}_{M} & =\frac{1}{2}\left[\left\langle M \bar{u}^{t}, M \bar{u}^{t}\right\rangle+\left\langle M \bar{v}^{t}, M \bar{v}^{t}\right\rangle\right] .
\end{aligned}
$$

Relationship (31) shows that the ASSA scheme conserves $\mathcal{E}$ in the limit of small time steps $\varepsilon \rightarrow 0$ and conserves the energy of the four-point averaged fields in the "quasigeostrophic" limit $\varepsilon \rightarrow \infty$. The damping term $2 \varepsilon f\left(\mathcal{K}-\mathcal{K}_{M}\right)$ is scale selective: it preferentially removes energy from the states with strong grid-scale components in the velocity field.

\section{d. Numerical testing}

In this section we present the results from five numerical codes: a standard explicit $\mathrm{C}$ grid (SEC) code, 2D implementations of the SSA and ASSA schemes, the OPA (Madec et al. 1999) ocean general circulation model, and the 3D baroclinic version of the ASSA algorithm.

In the first series of experiments we compared the performance of the SEC scheme (1)-(3) with ASSA
(18)-(20) using the following setup. The 2D shallowwater model was integrated for 50 days in a closed flatbottomed rectangular $4000 \mathrm{~km} \times 4000 \mathrm{~km}$ basin on the $f$ plane with a grid step of $100 \mathrm{~km}$. The parameters of the model experiment were the following: the basin depth $H=1 \mathrm{~km}, f=10^{-4} \mathrm{~s}^{-1}$, the reduced gravity $g$ $=0.1 \mathrm{~cm} \mathrm{~s}^{-2}$, and the bottom drag coefficient $\varepsilon=10^{-6}$ $\mathrm{s}^{-1}$. These values may be considered as typical for the high-latitude seas, where the internal Rossby deformation radius $(R=10 \mathrm{~km})$ is often unresolved even by high-resolution global OGCMs. Circulation within the box was forced by a steady zonal wind of the form $\tau_{x}$ $=\tau_{0} \sin \pi x / L \sin \pi y / L$, where $-L / 2<x, y<L / 2$ and $\tau_{0}=2 \mathrm{dyn} \mathrm{cm}^{-2}$. The time stepping of $1 \mathrm{~h}$ was used to integrate both the SEC and ASSA codes. As an additional benchmark we have also integrated the SSA code (9)-(11) for comparison.

Figure 1 shows the results of integration. As we see, the grid-scale noise present in the divergence field produced by the SEC scheme (left) is absent in the result of integration by the SSA algorithm, whose dispersion relation does not contain the troublesome factor $c$ before the Coriolis parameter. The divergence field (middle) is also virtually free of the grid-scale noise, which has been removed by the grid-scale damping property of the ASSA scheme.

Regarding the computational cost of the 2D setting, the SSA code requires 4.3 times more CPU time (and approximately 3 times more memory) than the SEC scheme. The ASSA algorithm consumed only $15 \%$ more CPU time with no extra memory requirements compared to SEC. These figures are valid for the versions of the SSA and ASSA algorithms with explicit representation of the pressure gradient terms, which give virtually the same results for the present setting as the patterns generated by the semi-implicit versions of these algorithms shown in Fig. 1.

In the second series of experiments we have checked the performance of the ASSA scheme against linear solutions of a model with conventional C-grid approximation (OPA OGCM; Madec et al. 1999). The 3D linear version of the ASSA code and OPA were configured 


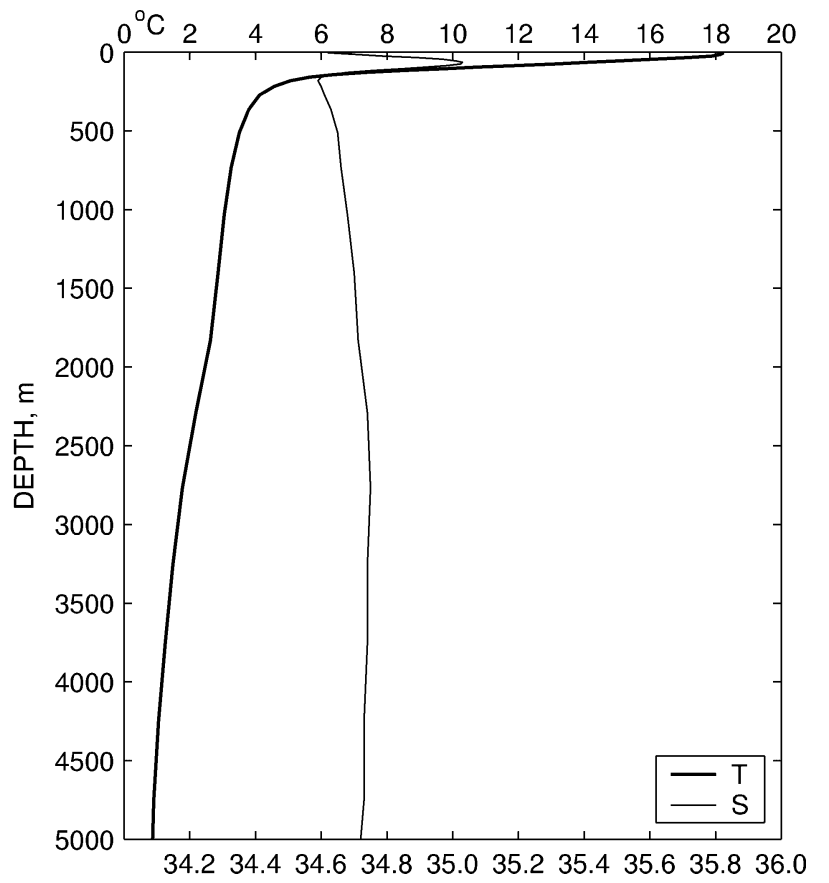

FIG. 2. Vertical temperature and salinity profiles used for initialization of the 3D OPA and ASSA models.

in a rectangular basin of constant depth $(H=5000 \mathrm{~m})$ $36^{\circ} \times 11^{\circ}$ in size. The basin was centered at the latitude $\varphi=40^{\circ} \mathrm{N}$ (Fig. 1). The initial conditions were specified as horizontally homogeneous temperature and salinity distributions, whose profiles are shown in Fig. 2. The corresponding first baroclinic deformation radius $R$ is $15 \mathrm{~km}$. We used the default 31-level vertical grid from OPA's documentation (Madec et al. 1999) and horizontal grid stepping of $\delta x=0.5^{\circ}$. The "wave resolution factor" of Adcroft et al. (1999) was $r=R / \delta x \sim 0.27$. Both models were integrated for 400 days from the state of no motion with a time step of $1 \mathrm{~h}$. The circulation was forced by the steady wind of the form

$$
\begin{aligned}
\tau_{x}(\varphi) & =\tau_{0} \sin \left[\pi\left(\varphi-40^{\circ}\right) / 13^{\circ}\right] \\
\tau_{0} & =0.1 \mathrm{dyn} \mathrm{cm}^{-2},
\end{aligned}
$$

which induces a weak anticyclonic single-gyre circulation in the domain. Such shape of the wind forcing generates Ekman downwelling with typical vertical velocities of $0.5-1 \mathrm{~m} \mathrm{yr}^{-1}$ with an exception of thin boundary layers where water is forced to rise by the continuity constraint. These boundary layers serve as a strong source of grid-scale noise in the conventional C-grid approximation.

The two upper panels in Fig. 3 show the vertical velocity field at $z=20 \mathrm{~m}$ after 400 days of integration of the OPA OGCM. The grid-scale noise generated by the conventional scheme appears to be so intense that we were forced to display the same solution after lowpass filtering in the meridional direction (Fig. 1, middle). On the contrary the noise is apparently absent in the simulation driven by the ASSA scheme (lower panel).

\section{Discussion}

Consideration of the symmetrized shifted approximation and development of the ASSA scheme was motivated by ill conditioning of the four-point averaging operator that acts on horizontal velocities present in the Coriolis terms of a GCM formulated on a $\mathrm{C}$ grid. As a consequence, when Coriolis terms dominate the horizontal momentum balance, $\mathrm{C}$-grid solutions are prone to grid-scale noise, belonging to the null space of the averaging operator. In the proposed scheme, we first employ the technique of shifted approximations of the Coriolis terms in the momentum equations and then center the resulting velocity fields by averaging over the four shifted solutions.

We have demonstrated that the new scheme has spectral properties of the inertia-gravity waves similar to those on the $\mathrm{B}$ and $\mathrm{C}$ grids at wavenumbers well below $2 \pi / \delta x$. At wavelengths close to the grid scale the ASSA scheme damps the transient inertia-gravity waves with a time scale proportional to $1 / f^{2} \delta t$. The damping is a payoff for the absence of extra computational modes and for the necessity to keep in memory the shifted solutions of the SSA method.

Similar to the B- and C-/D-grid approaches, the ASSA and SSA schemes can be readily utilized for implicit treatment of the Coriolis terms, since the pointwise linear subsystems of the shifted approximations can be inverted analytically. It should be noted that although we have used a semi-implicit scheme for approximation of the Coriolis terms, one could equally well use any explicit SSA scheme (e.g., fourth-order Runge-Kutta) on the four shifted equations and the gridscale noise issue will still be fixed by averaging over the four shifted solutions. We utilized the Crank-Nicholson scheme for illustrative purposes only, in order to demonstrate the ability of SSA and ASSA schemes to treat the Coriolis terms implicitly.

The ASSA scheme has a number of important features in representation of the Coriolis terms on the $\mathrm{C}$ grid:

1) it preferentially damps the grid-scale noise without artifical dissipation terms as it was done, for example, in the approach of Smith et al. (1990);

2) the method allows us to treat the Coriolis terms semiimplicitly and is easily extended to implement a general forward-backward scheme (Ames 1969) for the inertia-gravity waves, like the "mixed" time-differencing technique proposed by Janjic and WiinNielsen (1977);

3 ) in contrast to the C-/D-grid approach of Adrcroft et al. (1999), no additional degrees of freedom are introduced into the model variables;

4) the scheme allows us to solve shallow-water equations in the rigid-lid approximation.

At present we have successfully implemented the 

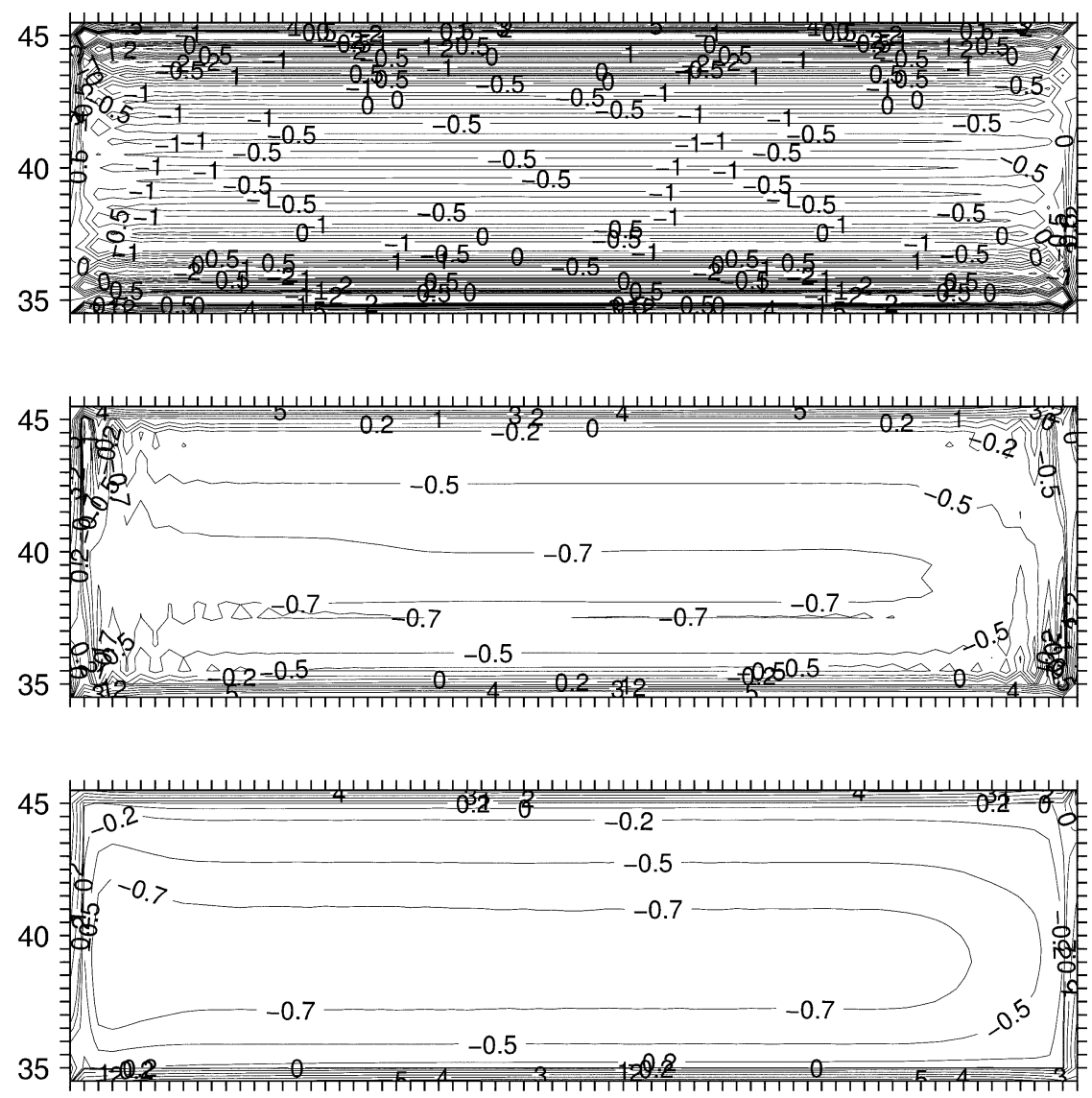

FIG. 3. Vertical velocity field after 400-day integration of the (top) OPA OGCM and (bottom) the same field obtained by using the ASSA scheme. (middle) The OPA solution low-pass filtered in the meridional direction. Units are in meters per year.

ASSA scheme in construction of an OGCM with implicit treatment of the barotropic mode under both rigid-lid and free-surface formulations. Preliminary experiments show remarkable robustness of the horizontal divergence and vertical velocity fields in comparison with the output of the conventionally formulated OPA OGCM. We believe that the proposed scheme is capable of improving the performance of C-grid models in applications with relatively coarse spatial and temporal resolutions.

Acknowledgments. This study was supported by the by the Office of Naval Research Grant NR00-95906 and by the Frontier Research System for Global Change through its sponsorship of the International Pacific Research Center. Two anonymous reviewers were extremely helpful with corrections and suggestions to the manuscript. Helpful discussions with Prof. V. Zalesnyy and Dr. A. Krylov are also acknowledged.

\section{REFERENCES}

Adcroft, A. J., C. N. Hill, and J. C. Marshall, 1999: A new treatment of the Coriolis terms in C-grid models at both high and low resolutions. Mon. Wea. Rev., 127, 1928-1936.
Ames, W. F., 1969: Numerical Methods for Partial Differential Equations. Nelson, $291 \mathrm{pp}$.

Arakawa, A., and V. R. Lamb, 1977: Computational design of the basic numerical processes of the UCLA general circulation model. Methods in Computation Physics, J. Chang, Ed., Academic Press, 174-267.

Delecluse, P., and V. B. Zalesnyy, 1996: On the numerical simulation of equatorial dynamics. Oceanology, 36, 22-37.

Janjic, Z., and A. Wiin-Nielsen, 1977: On geostrophic adjustement and numerical procedures in a rotating fluid. J. Atmos. Sci., 34, 297-310.

Madec, G., P. Delecluse, M. Imbard, and C. Lévy, 1999: OPA 8.1 Ocean General Circulation Model. Reference Manual, Institut Pierre-Simon Laplace, 91 pp.

Marshall, J., A. Adcroft, C. Hill, L. Perelman, and C. Heisey, 1997: A finite-volume incompressible Navier-Stokes model for studies of the ocean on parallel computers. J. Geophys. Res., 102, 57335752.

Smith, R. D., D. B. Boudra, and R. Bleck, 1990: A wind-driven isopycnal coordinate model of the north and equatorial Atlantic Ocean. 2: The Atlantic-basin experiments. J. Geophys. Res., 95 (C8), 13 105-13 128.

Winninghoff, F. J., 1968: On the adjustment toward a geostrophic balance in a simple primitive-equation model with application to the problems of initialization and objective analysis. Ph.D. thesis, University of California, Los Angeles, $161 \mathrm{pp}$. 
Copyright of Monthly Weather Review is the property of American Meteorological Society and its content may not be copied or emailed to multiple sites or posted to a listserv without the copyright holder's express written permission. However, users may print, download, or email articles for individual use. 